\title{
Presencia de metales pesados y semimetales en musgo en torno a la Estación Antártica Ecuatoriana Pedro Vicente Maldonado
}

\author{
DEISY SARMIENTOa, RONNY FLORES ${ }^{a}$ * \\ (a) Laboratorio de Química Ambiental, Facultad de Ciencias Químicas, \\ Universidad Central del Ecuador (UCE), Quito, Ecuador \\ *Correspondencia: raflores@uce.edu.ec
}

Recibido: 25 de marzo de 2014, Aceptado: 14 de agosto de 2014

\section{Resumen}

Durante la XV expedición ecuatoriana a la Antártida, desarrollada por el Instituto Antártico Ecuatoriano en los meses de febrero y marzo de 2011 , se recolectaron muestras de musgo Saniona Uncinata en el área cercana a la Estación Pedro Vicente Maldonado, ubicada en la Isla Greenwich, para conocer la posible afectación que tiene la actividad humana en la región. El musgo fue utilizado como biomonitor de la contaminación por metales pesados y semimetales. Se establecieron cinco puntos de muestreo que corresponden a áreas de influencia directa e indirecta de la Estación. Los elementos se cuantificaron por espectroscopia de absorción atómica y se determinó que el arsénico, cromo, plomo y cadmio se encuentran en mayor concentración en el musgo recogido en el área cercana al Módulo de los Generadores Eléctricos de la estación, que en el musgo recolectado de los otros sitios más distantes. Las actividades realizadas en el Módulo de los Generadores, como quema de combustibles fósiles, uso de pinturas anticorrosivas, procesos de soldadura, etc., pueden ser los causantes del incremento de metales en el sitio.

Palabras clave: musgo, contaminación, metales, Antártida, biomonitor.

\section{Presence of heavy metals and semimetals in moss that surrounds the Ecuadorian Antarctic Station Pedro Vicente Maldonado}

\section{Abstract}

During 15th Ecuadorian expedition to the Antarctic, carried out by the Antarctic Ecuadorian Institute during the months of February and March 2011, samples of moss Saniona Uncinata were collected in the area closest to the Pedro Vicente Maldonado Station, on the Greenwich Island, with the purpose of knowing how much human activity had affected the area. The moss was used as a bio-monitor of the contamination caused by heavy metals and semimetals. Five areas of sampling were established, corresponding to areas of direct or indirect influence of the Station. The elements were quantified by use of an atomic absorption spectroscopy. It was determined that arsenic, chrome, lead, and cadmium were found in greater percentages in the moss retrieved in the area close to the Generators Module of the Station than was found in samples from more distant sites. Activities proper to the area of the Generators Module, that is, burning of fossil fuel, use of anti-corrosive paint, soldering, among others, could be responsible for the increase of metals in the area.

Key words: moss, pollution, metals, Antarctica, biomonitor. 


\section{Introducción}

La Estación Científica Ecuatoriana Pedro Vicente Maldonado está ubicada en la Punta Fort William de la Isla Greenwich que pertenece al archipiélago Shetland del Sur a $62^{\circ} 26^{\prime} 57,6^{\prime \prime}$ de latitud sur y $59^{\circ} 44^{\prime} 32,1^{\prime \prime}$ de longitud oeste. Es una base antártica temporal que funciona durante el verano austral que empieza a finales de diciembre y se extiende hasta marzo del siguiente año. Tiene una capacidad aproximada para treinta personas y las labores que se realiza en ella son investigativas en los campos de geología, meteorología, oceanografía, contaminación ambiental y fisiología humana.

La Antártida, a pesar de ser el continente más aislado, no está libre de la contaminación por el ser humano. La entrada antropogénica de metales pesados en el ambiente antártico puede ser originada tanto de fuentes locales como globales. En las fuentes locales se incluyen estaciones científicas, incineración de basura, uso de combustibles y pinturas y aguas residuales. Mientras que las fuentes globales están relacionadas con el transporte atmosférico de elementos procedentes de latitudes más bajas [1].

Para determinar metales y semimetales presentes en el ambiente antártico, puede emplearse musgo como biomonitor o bioindicador. Un bioindicador es un ser vivo que manifiesta una respuesta clara frente a una determinada sustancia y a una determinada concentración de esa sustancia. Existen algunos vegetales que son buenos bioindicadores de la contaminación atmosférica, como los musgos y los líquenes, no por los efectos que sobre ellos se producen sino porque son bioacumuladores, pues absorben los contaminantes directamente del aire con una perfecta correlación entre la concentración en la atmósfera y concentración en el ser vivo [2]. Estos organismos acumulan específicamente metales pesados en sus células en concentraciones superiores a las que se dan en los medios donde habitan. La concentración de metales pesados en el ambiente es más fácil de detectar a través del musgo que si se mide directamente del aire o del agua en que viven [3].

Las briofitas han sido ampliamente utilizadas como bioindicadores de la contaminación atmosférica en la Antártida por su presencia dominante en áreas libres de nieve y su facilidad de muestreo [2]. El musgo es la especie vegetal que cubre el $9 \%$ del área total alrededor de la estación ecuatoriana Pedro Vicente Maldonado [4].
Los elementos que se analizaron, por su toxicidad y uso generalizado fueron $\mathrm{As}, \mathrm{Cr}, \mathrm{Hg}, \mathrm{Pb}$ y $\mathrm{Cd}$ [5]. El uso de combustibles fósiles, equipos y maquinarias pueden ser los causantes de la presencia de estos metales en la Antártida. El arsénico puede provenir de la quema de combustibles fósiles o conservantes de madera. El cromo de su empleo en recubrimientos metálicos y pinturas anticorrosivas. El mercurio se libera durante la quema de combustibles fósiles, está presente en baterías viejas y lámparas fluorescentes. El plomo se utiliza en soldaduras, pinturas y dispositivos electrónicos (televisores, computadoras, celulares y demás). El cadmio se emplea en recubrimiento de metales, baterías recargables, equipos electrónicos y pigmentos; y se libera con la quema de carbón [6]. Además, la contaminación antropogénica puede llegar desde África, Sudamérica y Australia transportada por la atmósfera [7].

La presente investigación es parte del proyecto C- 02 11: Presencia de contaminantes antropogénicos orgánicos volátiles, orgánicos persistentes, gases invernadero y metales pesados en torno de la estación ecuatoriana Pedro Vicente Maldonado, que se ejecuta con el apoyo del Instituto Antártico Ecuatoriano.

\section{Parte experimental}

\section{Recolección y pretratamiento de las muestras}

Durante la tercera etapa de la XV expedición ecuatoriana a la Antártida, en los meses de febrero y marzo de 2011, se recolectaron muestras de musgo Saniona Uncinata. Se establecieron cinco puntos de muestreo, que corresponden a las áreas de influencia directa e indirecta de la estación Pedro Vicente Maldonado [8] 1500 m y 5000 m alrededor de la estación, respectivamente, Fig. 1). En cada punto se tomaron tres muestras de musgo (50 g aprox.) (Fig. 2). Las muestras se secaron en una estufa eléctrica a $80^{\circ} \mathrm{C}$ durante toda la noche, se almacenaron herméticamente y se transportaron, en refrigeración, hasta Ecuador a la Facultad de Ciencias Químicas de la Universidad Central del Ecuador. Los sitios de recolección de las muestras de musgo fueron georeferenciados para poder volver a muestrear en los mismos sitios en expediciones futuras, lo que permitirá monitorear la evolución de la concentración de los contaminantes. 


\section{Análisis de metales y semimetales}

En las muestras recolectas se separó cuidadosamente el suelo del musgo con un tamiz de $630 \mu \mathrm{m}$. El musgo se sometió a digestión, en un digestor de microondas marca Berghof, modelo Speedwave Four, según una modificación del método Microwave Digestion of Herbs, incluido en el equipo. A $1000 \mathrm{mg}$ de muestra se añadió $12 \mathrm{~mL}$ de ácido nítrico $65 \%$ y $4 \mathrm{~mL}$ de peróxido de hidrógeno $30 \%$. Se digestó durante 10 minutos a $170^{\circ} \mathrm{C}$ con una presión de 80 bar y $80 \%$ de potencia. Todos los reactivos fueron grado analítico. Las muestras se analizaron por triplicado.

Los elementos extraídos se cuantificaron por espectroscopia de absorción atómica a la llama, según los métodos EPA 7061A para arsénico, 7470A para mercurio, 7130 para cadmio, 7420 para plomo y 7190 para cromo, en un equipo marca Perkin Elmer, modelo AANALYST 100. Todos los análisis se realizaron en un laboratorio acreditado perteneciente a la Facultad de Ciencias Químicas.

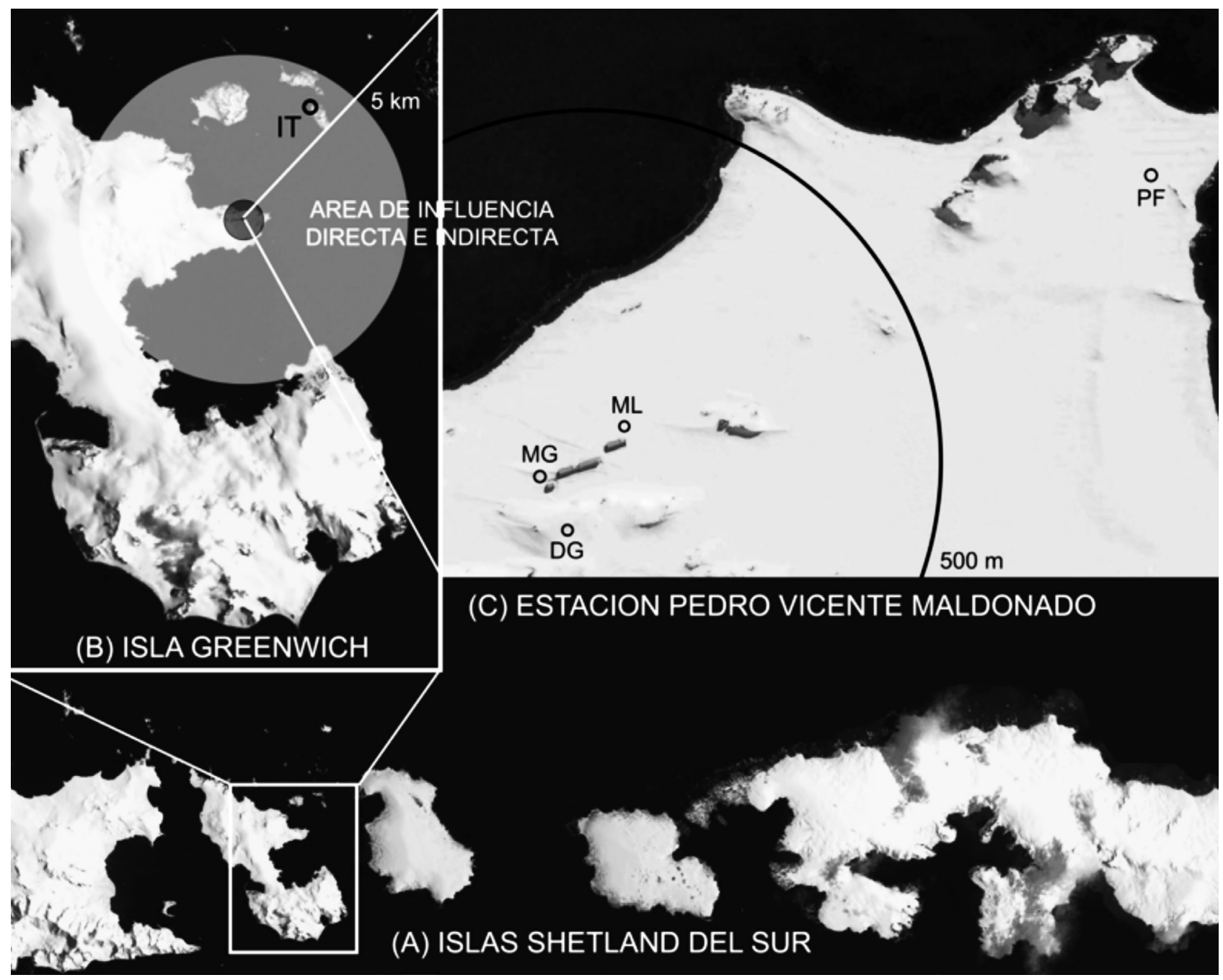

Figura 1. (A) Fotografía satelital de las Islas Shetland del Sur en la Península Antártica. (B) Isla Greenwich donde se indican las áreas de influencia directa e indirecta de la estación y el sitio de muestreo IT en la Isla Torre. (C) Estación Científica Ecuatoriana Pedro Vicente Maldonado en Punta Fort William, se indica el área de influencia directa y los puntos de muestreo MG frente al módulo de generadores, DG detrás del módulo de generadores, ML frente al módulo de laboratorio y PF punta Figueroa. Foto satelital tomada de Landsat Image Mosaic of Antarctica (LIMA), (http:// lima.usgs.gov/l, modificada por R. Flores (2012). 


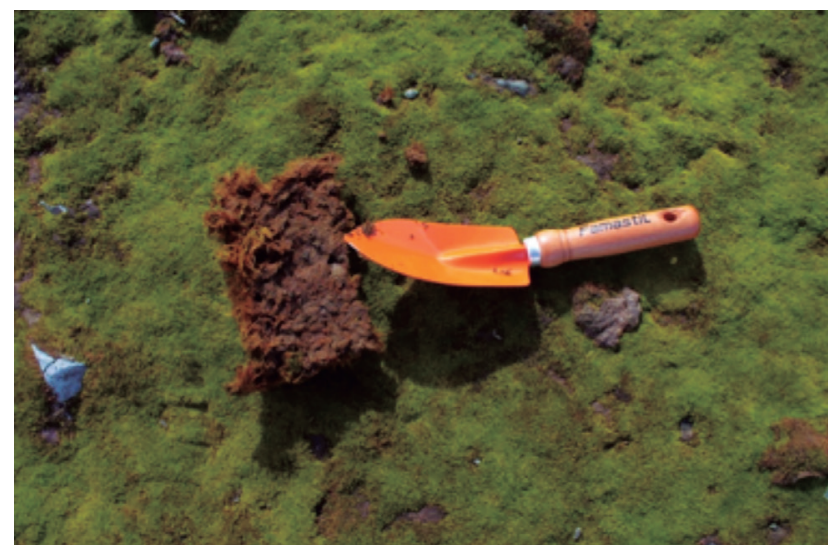

Figura 2. Toma de muestra de musgo en la Antártida. Foto R. Flores (2011)

\section{Resultados y discusión}

Los sitios de muestreo fueron cinco distribuidos entre el área de influencia directa e indirecta de la estación (Fig. 1). El área de influencia directa es el ámbito geográfico donde se presenta de manera evidente los impactos ambientales. Para la estación es el espacio físico ocupado en forma permanente por los diferentes módulos, las operaciones que allí se realizan y las actividades de investigación científica. Incluye todos los sitios donde se depositan materiales excedentes, lugares de muestreo y mo- nitoreo. Esta área es susceptible de afectación por las diferentes actividades, tanto de construcción como de mantenimiento y operación, que cotidianamente se llevan a cabo en la estación. El área de influencia directa comprende las instalaciones de la estación hasta una distancia aproximada de 500 metros alrededor de la misma [8].

El área de influencia indirecta de la estación es la zona sobre la cual uno o varios aspectos ambientales afectados en el área de influencia directa, pueden, a su vez, trasladar esas afectaciones, aunque sea en mínima proporción, a otros aspectos ambientales más alejados de las actividades directas de la estación. El área de influencia indirecta cubre la Punta Fort William, incluyendo los ecosistemas terrestres de las islas Dee, Barrientos y Torre, además, de los cuerpos hídricos de Ensenada Guayaquil y Bahía Chile, hasta una distancia de cinco kilómetros alrededor de la estación [8].

Los puntos $M G, M L$ y $D G$ corresponden al área de influencia directa (Tabla 1 y Fig. 1). Los puntos IT y PF comprenden el área de influencia indirecta. Para el análisis estadístico de resultados se utilizó la Prueba de Rango Múltiple de Duncan, que es una comparación de las medias de todos los tratamientos al $95 \%$ de confianza con el software STATISTICA 8.0.

Tabla 1. Sitios donde se tomaron las muestras de musgo en torno a la Estación Científica Ecuatoriana Pedro Vicente Maldonado. MG, ML y DG corresponden a área de influencia directa, PF e IT a área de influencia indirecta.

\begin{tabular}{cllc}
\hline CLAVE & Nombre del sitio & Georeferencia, UTM & $\begin{array}{c}\text { Distancia desde la } \\
\text { Estación }\end{array}$ \\
\hline MG & Módulo de generadores & $21 \mathrm{E}, 358524 \mathrm{X}, 3072780 \mathrm{Y}$ & $14 \mathrm{~m}$ \\
ML & Módulo de laboratorio & $21 \mathrm{E}, 358617 \mathrm{X}, 3072849 \mathrm{Y}$ & $25 \mathrm{~m}$ \\
DG & Detrás de los generadores & $21 \mathrm{E}, 358565 \mathrm{X}, 3072720 \mathrm{Y}$ & $52 \mathrm{~m}$ \\
PF & Punta Figueroa & $21 \mathrm{E}, 359283 \mathrm{X}, 3073205 \mathrm{Y}$ & $828 \mathrm{~m}$ \\
IT & Isla Torre & $21 \mathrm{E}, 358677 \mathrm{X}, 3077012 \mathrm{Y}$ & $4234 \mathrm{~m}$ \\
\hline
\end{tabular}

El arsénico tuvo una concentración diez veces mayor en MG que en el resto de sitios de muestreo, IT y PF presentaron las más bajas concentraciones de arsénico (Fig. 3). El cadmio (Fig. 4) mostró una concentración alta en todos los puntos excepto en IT que presentó la concentración más baja. El cromo 
(Fig. 5) tuvo una concentración alta en MG y ML y la más baja en PF. El mercurio (Fig. 6) presentó una concentración alta en $M G$ y $M L$, siendo la más alta en ML, y la más baja en PF. El plomo (Fig. 7) estuvo en mayor concentración en MG y en menor en DG. $\mathrm{Pb}$ y $\mathrm{Hg}$ fueron los metales que se encontraron en mayor concentración durante el estudio y corresponden a los módulos de generadores y laboratorio. MG tiene las más altas concentraciones para $\mathrm{As}, \mathrm{Cd}, \mathrm{Cr}$ y Pb; en cambio IT y PF tienen, en general, las concentraciones más bajas para la mayoría de contaminantes. Las bajas concentraciones en los puntos IT y PF se explica ya que están localizados en la zona de influencia indirecta, por lo que no están muy afectados por la contaminación directa generada en la estación.

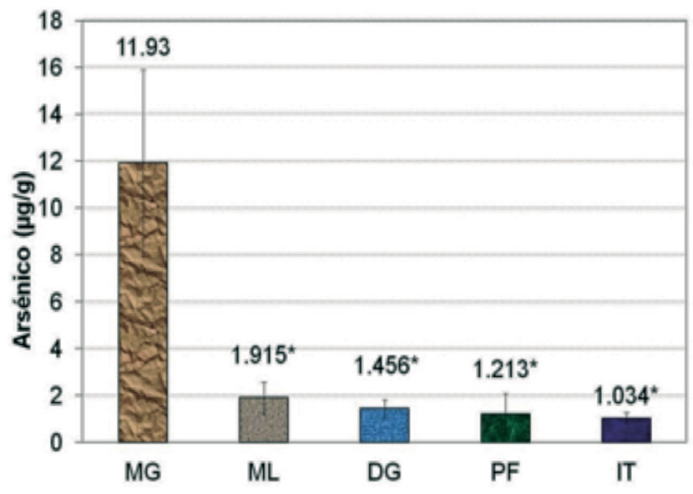

Figura 3. Concentración de arsénico en un gramo de musgo seco en los cinco puntos de muestreo. La concentración de arsénico es diez veces mayor en MG que en los otros sitios de muestreo y las concentraciones en los restantes cuatro puntos son similares entre sí. Barra de error corresponde a la desviación estándar de tres determinaciones. $\left(^{*}\right)$ Indica que no hay diferencia significativa entre medias de concentración con la prueba de Rango Múltiple Duncan al 95\% de confianza.

En cambio, MG es el sitio donde se realiza la mayoría de actividades de mantenimiento de la estación, además, allí funcionan los generadores por lo que fue el módulo que presentó mayor contaminación. $M L$, es el segundo módulo, después de $M G$, con contaminación más alta, hay que recordar que este módulo fue recientemente inaugurado, por lo que cabría esperar que el proceso de construcción fue el causante del alto nivel de contaminación en el sitio.

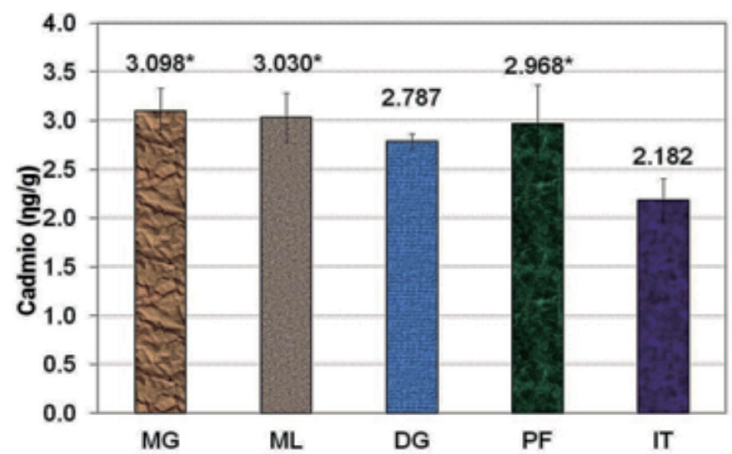

Figura 4. Concentración de cadmio en un gramo de musgo seco en los cinco puntos de muestreo. El cadmio tiene una concentración parecida en la mayoría de sitios analizados, excepto en IT que es la más baja. Barra de error corresponde a la desviación estándar de tres determinaciones. (*) Indica que no hay diferencia significativa entre medias de concentración con la prueba de Rango Múltiple Duncan al 95\% de confianza.

El arsénico es un elemento ubicuo que puede hallarse en matrices bióticas y abióticas y es movilizado a través del ambiente por procesos naturales y antropogénicos [9]. La alta concentración de arsénico en $M G$ con respecto a los otros puntos de muestreo se asocia, sin duda, a la actividad humana. El arsénico puede provenir de la quema de combustibles fósiles o conservantes de madera. Concentraciones importantes de arsénico se han encontrado en macroalgas antárticas, 41.0 a $447 \mu \mathrm{g} / \mathrm{g}$ peso seco [10]; las algas toman el arsénico de especies en solución en el agua del mar, en cambio, el musgo lo toma directamente del ambiente.

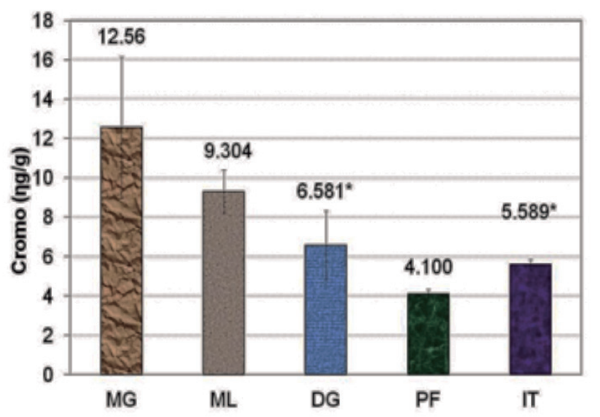

Figura 5. Concentración de cromo en un gramo de musgo seco en los cinco puntos de muestreo. El cromo está presente en mayor concentración en MG y ML. Y las concentraciones entre IT y DG son similares. La menor concentración de cromo se encontró en PF. Barra de error corresponde a la desviación estándar de tres determinaciones. (*) Indica que no hay diferencia significativa entre medias de concentración con la prueba de Rango Múltiple Duncan al 95\% de confianza. 
Las actividades humanas como industria, disposición de aguas residuales y el uso de combustibles fósiles cambian los niveles naturales de $\mathrm{Hg}$. Santos et al. [1] encontraron $\mathrm{Hg}$ en briofitas en una concentración de 23.1 a $39.5 \mathrm{ng} / \mathrm{g}$, concentraciones que fueron consideradas bajas. En este estudio la concentración de $\mathrm{Hg}$ estuvo en el orden de los microgramos, concentración mil veces mayor a lo determinado por Santos. La presencia de mercurio en matrices bióticas sugiere que se está produciendo bioacumulación debido a su alta movilidad y afinidad por materia orgánica. El mercurio se libera durante la quema de combustibles fósiles, de baterías viejas y lámparas fluorescentes.

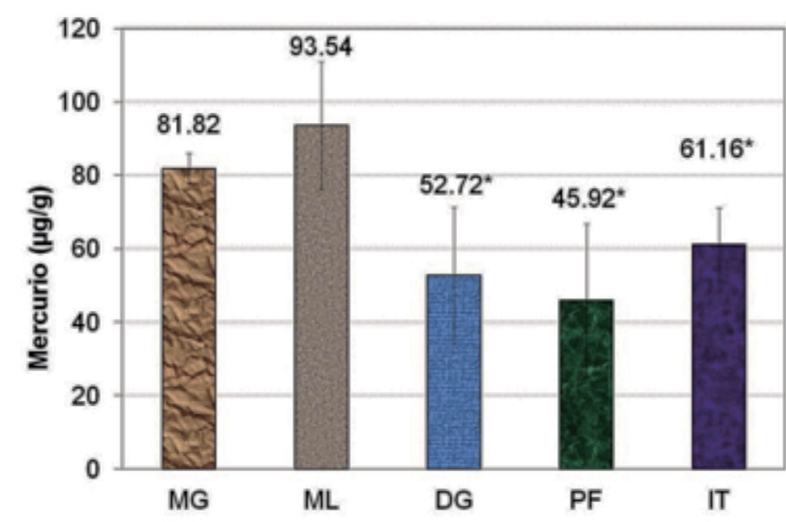

Figura 6. Concentración de mercurio en un gramo de musgo seco en los cinco puntos de muestreo. El mercurio está en una concentración mayor en MG y ML. DG, IT y PF tienen concentraciones bajas y parecidas. Barra de error corresponde a la desviación estándar de tres determinaciones. (*) Indica que no hay diferencia significativa entre medias de concentración con la prueba de Rango Múltiple Duncan al 95\% de confianza.

La presencia de plomo se puede asociar al uso de pinturas, equipos de soldadura y a la existencia de baterías usadas. Webster et al. [11] llegaron a relacionar la concentración elevada de plomo en el suelo de una antigua estación antártica con una pintura roja utilizada en la estación.

La presencia de cromo puede deberse a su empleo en recubrimientos metálicos y pinturas anticorrosivas y el cadmio a su uso en recubrimiento de metales, baterías recargables y pigmentos. En la estación ecuatoriana, las actividades realizadas en el módulo de los generadores como uso de combustibles, equipos y maquinarias pueden ser las causantes del incremento de metales en este sitio.
Otras investigaciones relacionadas con la contaminación por metales en la Antártida [1], [12] y [13] han determinado presencia de metales como cadmio $(0,13-0,07 \mu \mathrm{g} / \mathrm{g})$, mercurio $(23,1 \eta \mathrm{g} / \mathrm{g})$, plomo $(0,01-0,03 \mu \mathrm{g} / \mathrm{g})$ y cromo (menor a $0,03 \mu \mathrm{g} / \mathrm{g}$ ) en algas, musgos (especies distintas a las utilizadas en esta investigación), pingüinos y hielo lo que confirma que la Antártida a pesar de ser un continente aislado, sufre contaminación ambiental ya sea por arrastre a través del aire o agua y/o por actividades en el sitio.

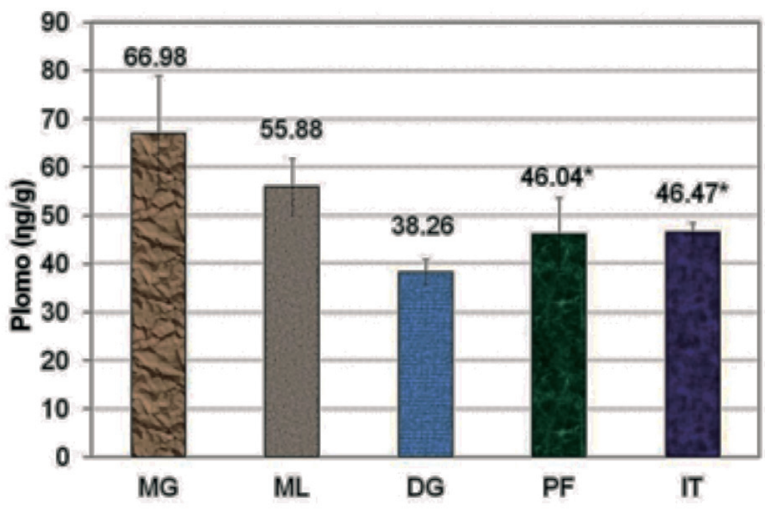

Figura 7. Concentración de plomo en un gramo de musgo seco en los cinco puntos de muestreo. El plomo está presente en mayor concentración en MG. IT y PF tienen concentraciones similares. La concentración más baja se localizó en DG. Barra de error corresponde a la desviación estándar de tres determinaciones. (*) Indica que no hay diferencia significativa entre medias de concentración con la prueba de Rango Múltiple Duncan al 95\% de confianza.

\section{Conclusiones}

De los resultados del estudio se concluye que existe mayor presencia de metales y semimetales en el área de influencia directa que en el área de influencia indirecta ocasionada por la presencia de la estación Pedro Vicente Maldonado. El metal que se encuentra en mayor cantidad en todos los sitios de muestreo fue el mercurio. El $\mathrm{Hg}$ forma compuestos volátiles, como el dimetilmercurio, que escapa al ambiente por acción de bacterias anaerobias en los sedimentos y puede bioacumularse en el musgo que lo toma del aire por el arrastre del contaminante a través del aire y agua que llegan a la Antártida. En expediciones futuras a la Antártida se tomarán muestras de los productos utilizados en el módulo de generadores de la estación para poder determinar cuáles son las 
posibles fuentes de la contaminación encontrada y poder realizar recomendaciones sobre qué artículos deben prohibirse su uso en la Antártida. También, se volverá a tomar muestras de musgo en los mismos sitios lo que permitirá monitorear la evolución de la concentración de los contaminantes con el tiempo.

\section{Agradecimientos}

Los autores agradecen al Instituto Antártico Ecuatoriano (INAE) y a la Facultad de Ciencias Químicas de la Universidad Central del Ecuador por el apoyo brindado para la realización de esta investigación.

\section{Referencias}

[1] Santos I.R., E.V. Silva-Filho, C. Schaefer, et al. (2006). Escribe texto o la dirección de un sitio web, o bien, traduce un documento. Cancelar Alpha Baseline mercury and zinc concentrations in terrestrial and coastal organisms of Admiralty Bay, Antarctica. Environmental Pollution 140: 304-311.

[2] Yogui G.T., J.L. Sericano. (2008). Polybrominated diphenyl ether flame retardants in lichens and mosses from King George Island, maritime Antarctica. Chemosphere 73: 1589-1593.

[3] Bargagli R., D.H. Brown \& L. Nelli. (1995). Metal biomonitoring with mosses: procedures for correcting for soil contamination. Environmental Pollution 89 (2): 169-175.

[4 Ordóñez N., H. Yii, W. Cárdenas, et al. (2008). Estudio preliminar de la cobertura superficial en la Isla Greenwich Antártida. Revista Tecnológica ESPOL 21 (1): 17-21.

[5] Negri A., K. Burns, S. Boyle, et al. (2006). Contamination in sediments, bivalves and sponges of McMurdo Sound, Antarctica. Environmental Pollution 143: 456-467.

[6] Noriega P., A. Mediciz, A. Murillo, et al. (2008). Estudio de la concentración de cadmio y plomo en el aire de la ciudad de Quito, empleando briofitas como biomonitores. La Granja 8(2):17-24.

[7] Klánová J., N. Matykiewiczová, Z. Mácka, et al. (2008). Persistent organic pollutants in soils and sediments from James Ross Island, Antarctica. Environmental Pollution 152: 416-423.

[8] SAMBITO. (2011). (en línea). Estudio de impacto ambiental Ex-post Estación Científica Ecuatoriana Pedro Vicente Maldonado, Isla Greenwich-Shetland del Sur-Antártida. <http://www.slideshare.net/sambitoeco/eia-expost-pevima>. Acceso 21/03/2012.

[9] Vodopívez C., S. Farías, P. Smichowski, D. Vélez, et al. (2004). Arsénico total e inorgánico en macroalgas antárticas. Actas del Vo Simposio Argentino y $l^{\circ}$ Latinoamericano sobre Investigaciones Antárticas CD-ROM. Resumen expandido N. 205BG.

[10] Smichowski P., S. Farias, L. Valiente, et al. (2004). Total arsenic content of nine species of Antarctic macro algae as determined by electrothermal atomic absorption spectrometry. Analytical and Bioanalytical Chemistry 378: 465-469.

[11 Webster J., K. Webster, P. Nelson, et al. (2003). The behaviour of residual contaminants at a former station site, Antarctica. Environmental Pollution 123: 163-179.

[12] Bargali, R. (2008). Environmental contamination in Antartic ecosystems. Science of The Total Environment 400(1-3): 212-226.

[13] Szefer, P., Szefer, K., Pempkowiak, J., Skwarzec, B., y Bojanowski, R. (1994). Distribution and coassociations of selected metals in seals of the Antartic. Environmental Pollution 83(3): 314-349. 